\title{
Correction \\ Correction: Michler et al. Review and Assessment of the Effect of Hydrogen Gas Pressure on the Embrittlement of Steels in Gaseous Hydrogen Environment. Metals 2021, 11, 637
}

\author{
Thorsten Michler*(D), Ken Wackermann and Frank Schweizer
}

check for updates

Citation: Michler, T.; Wackermann, K.; Schweizer, F. Correction: Michler et al. Review and Assessment of the Effect of Hydrogen Gas Pressure on the Embrittlement of Steels in Gaseous Hydrogen Environment. Metals 2021, 11, 637. Metals 2021, 11, 1158. https://doi.org/10.3390/ met11081158

Received: 10 June 2021

Accepted: 7 July 2021

Published: 22 July 2021

Publisher's Note: MDPI stays neutral with regard to jurisdictional claims in published maps and institutional affiliations.

Copyright: (c) 2021 by the authors. Licensee MDPI, Basel, Switzerland. This article is an open access article distributed under the terms and conditions of the Creative Commons Attribution (CC BY) license (https:// creativecommons.org/licenses/by/ $4.0 /)$.
Fraunhofer Institute for Mechanics of Materials, Woehlerstrasse 11, 79108 Freiburg, Germany; ken.wackermann@iwm.fraunhofer.de (K.W.); frank.schweizer@iwm.fraunhofer.de (F.S.)

* Correspondence: thorsten.michler@iwm.fraunhofer.de

\section{Error in Figure 1}

In the original article [1], there was a mistake in Figure 1 as published. Figure $1 \mathrm{~b}$ is incorrect and must be deleted. The corrected Figure 1 appears below. The authors apologize for any inconvenience caused and state that the scientific conclusions are unaffected. The original article has been updated.

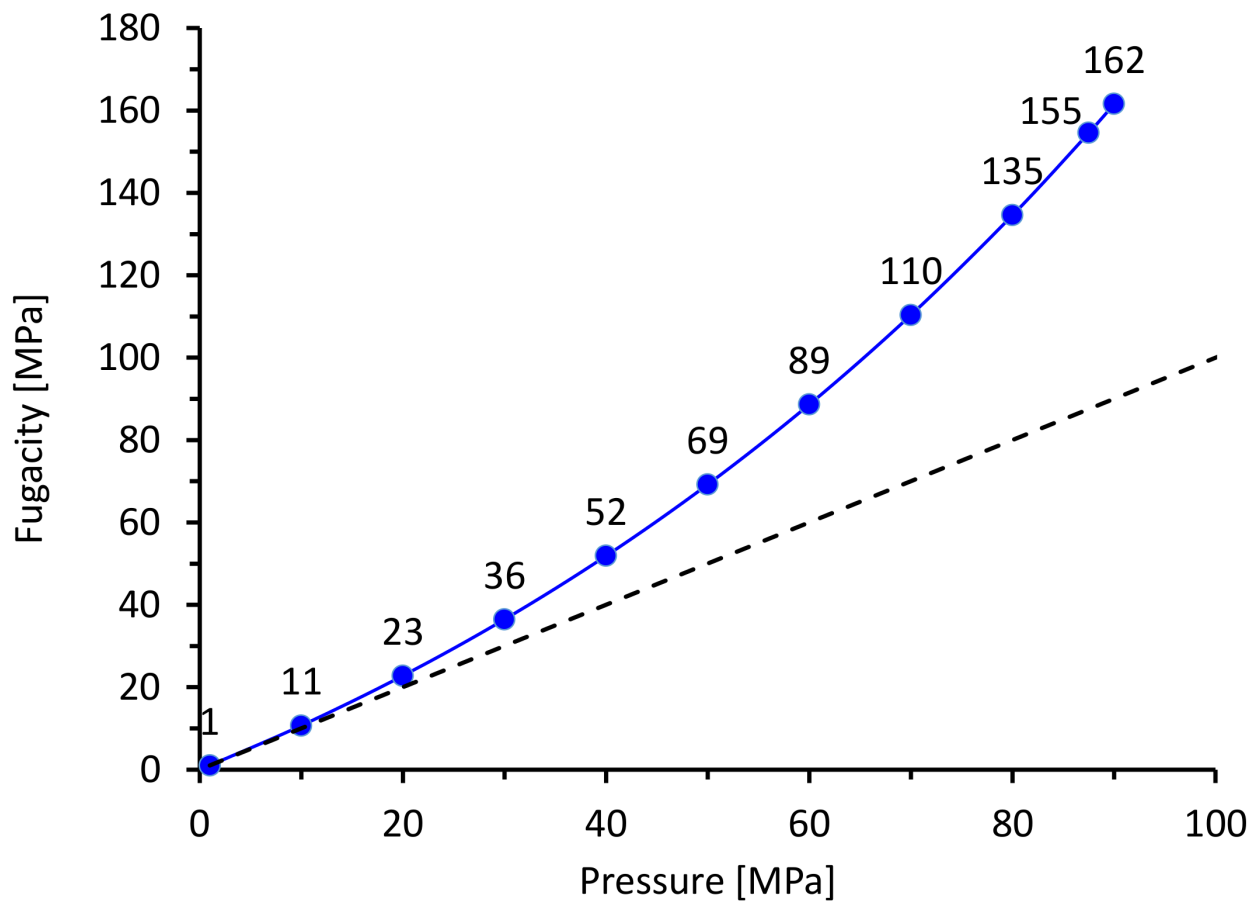

Figure 1. Correlation between pressure and fugacity at room temperature calculated by Equation (1).

\section{Error in Figure 5}

In the original article, there was a mistake in Figure 5 as published. Figure 5a is incorrect. The corrected Figure 5 appears below. The authors apologize for any inconvenience caused and state that the scientific conclusions are unaffected. The original article has been updated. 


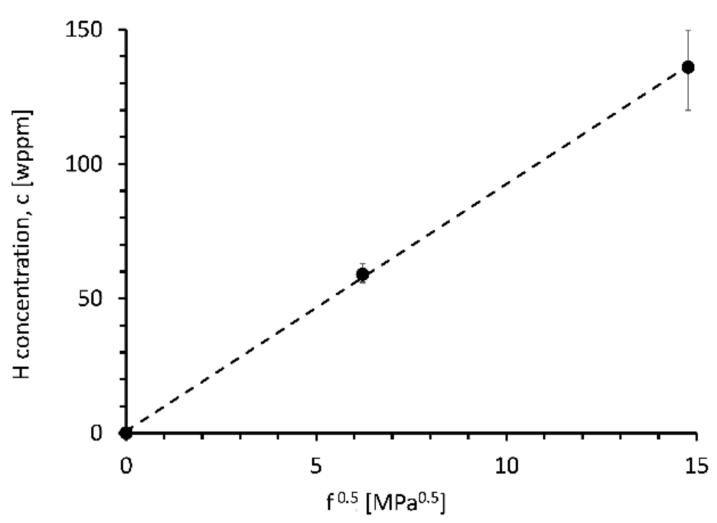

(a)

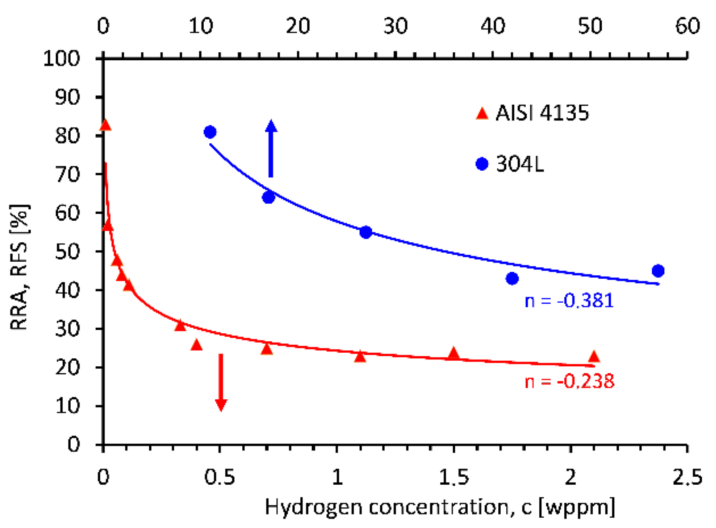

(b)

Figure 5. (a) Fugacity-modified Sievert's law with data from [50,51], (b) RRA of $304 \mathrm{~L}$ austenitic stainless steel [5] and relative fracture stress (RFS) of AISI 4135 martensitic high strength steel [7] pre-charged with hydrogen as a function of hydrogen concentration.

\section{Text Correction}

There was an error in the original article. When introducing the hydrogen fugacity, Sievert's law does not translate to $f^{0.46}$.

A correction has been made to Section 2: Experimental and analytical details, paragraph 3.

With $b=$ constant, $R=$ universal gas constant, and $T=$ temperature [9]. The correlation between pressure and fugacity is shown in Figure 1a. At room temperature, $f \approx p$ for pressures lower than $1 \mathrm{MPa}$, which is known as Sievert's law. For higher pressures, the non-ideal gas behavior must be considered especially in the power laws of rate limiting steps 3 and 4. Using Sievert's law as an example, $p^{0.5}$ translates to $f^{0.5}$ [9].

A correction has been made to Section 4: Discussion, paragraph 1.

As mentioned previously, the experimental data were fitted using Equation (2). For $|n|=0.5$, all rate limiting steps (Figure 4) would be undistorted. However, the analysis performed in this study shows that the influence of hydrogen fugacity upon the mechanical properties of steels does not follow a $f^{|0.5|}$ law for a wide variety of microstructures, strength levels, and test methods (Table 1).

A correction has been made to Section 4: Discussion, paragraph 5.

However, the analysis of data obtained with hydrogen pre-charged materials (Figure 5b) reveals $|n|$ values in the same range as obtained with tests in gaseous hydrogen atmosphere being significantly lower than 0.5 .

A correction has been made to Section 5: Summary, paragraph 3.

- The exponent $|n|$ was found to be smaller than 0.4. Since theoretical assumptions predict $n=0.5$ for a fully undistorted hydrogen reaction chain, the reviewed data are interpreted in a way that hydrogen-dislocation interactions are the rate-limiting step controlling hydrogen effects in steels.

The authors apologize for any inconvenience caused and state that the scientific conclusions are unaffected. The original article has been updated.

Conflicts of Interest: The authors declare no conflict of interest.

\section{Reference}

1. Michler, T.; Wackermann, K.; Schweizer, F. Review and Assessment of the Effect of Hydrogen Gas Pressure on the Embrittlement of Steels in Gaseous Hydrogen Environment. Metals 2021, 11, 637. [CrossRef] 